\title{
Paint It Black
}

Dear Reader,

Several confusing terms are currently going the rounds and "zero emission" is just one of them. It gives the impression that a mobility solution will soon become available which generates no emissions of any kind. This is clearly nonsense, if you think about it carefully. Every person produces emissions of heat, $\mathrm{CO}_{2}$ and methane that have no impact on the global climate. The only exception are the noise emissions that take the form of speech. The appropriate terms in this context are "climate neutrality" or "net zero.” We will achieve net zero when the quantity of $\mathrm{CO}_{2}$ that is emitted corresponds to the quantity that was stored in the past.

One source of the harmful noise emissions mentioned above is the discussion about the hydrogen combustion engine. The debate concerns whether or not an engine of this kind can be considered to be zero emission. From a purely technical perspective, this question is pointless, because it goes without saying that engines generate emissions. What we need to ask is whether it is net zero. The fuel contains no carbon, but the air that the engine takes in does. There are also other sources of greenhouse gas such as oils, which can make their way into the combustion chamber in the form of blow-by, and also the SCR system, where aqueous urea is converted into ammonia and $\mathrm{CO}_{2}$ and used to reduce the $\mathrm{NO}_{\mathrm{x}}$ emissions that are produced when the gas mixture cannot be made lean enough. But this $\mathrm{CO}_{2}$ was also previously stored. The gas that is released is first taken from the environment and then returned to it. All of this means that the engine can be regarded as climate neutral.

It would be a disaster if we were to regulate out of existence a technology that has significant potential for reducing emissions, precisely because it offers solutions for applications where defossilization would otherwise be difficult to achieve. A measurement taken at the tailpipe will show the presence of $\mathrm{CO}_{2}$, but deciding to ban these engines on this basis would simply be ridiculous.

I hope you enjoy this completely technology-neutral issue of MTZ.

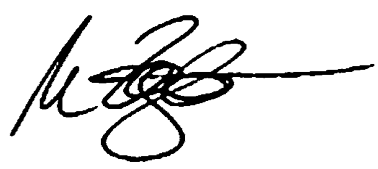

Marc Ziegler

Deputy Editor in Chief

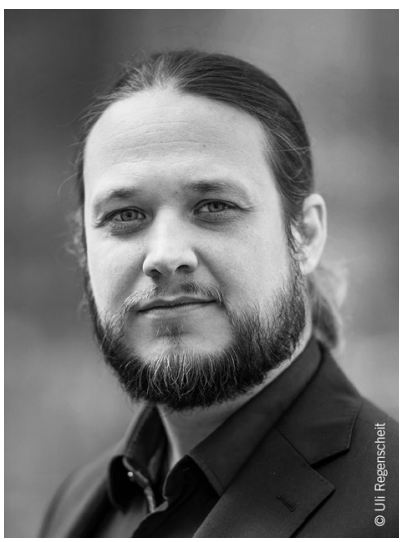

Consistent further development of the rotary shaft seal ElroSeal ${ }^{\mathrm{TM}}$ product family. Designed for high rotational movements of over $100 \mathrm{~m} / \mathrm{s}$, they reliably achieve high demands on leakage safety, pressures, temperatures, speeds and dry running.

Accelerate with us into the future

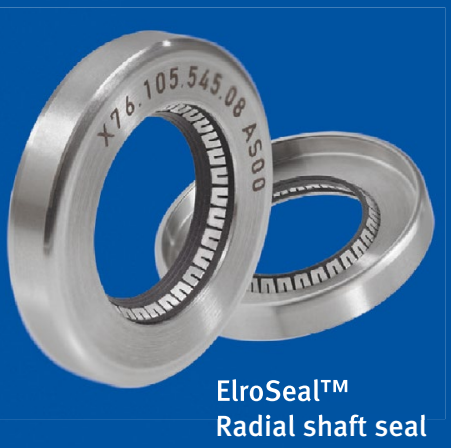

automotive@elringklinger.com Phone +49 7142 583-192 www.ek-kt.de/powertrain-electric 